\title{
Transient Activation in a Network of Coupled Map Neurons
}

\author{
J. M. Casado* \\ Área de Física Teórica, Universidad de Sevilla, Apartado Correos 1065, 41080, Sevilla, Spain
}

(Received 23 June 2003; published 10 November 2003)

\begin{abstract}
The focus of this Letter is on the activity of a network of neurons pairwise coupled by inhibitory connections. Each neuron is represented by a two-dimensional map capable, when isolated, of a rich variety of complex dynamical regimes. It is shown that the network exhibits a stimulus-dependent sequential activation and inactivation of subgroups of neurons. This complex behavior is rather similar to some spatiotemporal features observed in the first stages of the olfaction process in some insects and suggests the possibility of large scale simulation of these processes by using reasonable computational capabilities.
\end{abstract}

DOI: $10.1103 /$ PhysRevLett.91.208102

PACS numbers: 87.18.Sn, 05.45.Ra, 89.75.Hc

The understanding of how the sensory world is represented in the electrical activity of the brain is one of the fundamental tasks of neuroscience [1,2]. By now, it is well known that the information coming from sensory signals can be encoded into complex patterns of neuronal activity: each stimulus is represented by a specific and highly reproducible sequence of firing across some specific neurons $[3,4]$. To analyze the neuronal dynamics that gives rise to the odor-encoding capabilities of some invertebrates, networks of conductance-based model neurons have been developed which show such patterns of transient synchronization [5]. The aim of these studies has been to reproduce as close as possible the experimental results and so, systems of a great number of differential equations have been used to model each one of the neurons of the network. A price must be paid, however, for the use of such a detailed model. The high dimensionality of the dynamical system as well as the strong nonlinear character of its equations are significant obstacles for the understanding of the collective behavior of the network.

In this and other similar cases, the use of a simplified model of a neuron is advisable in order to be able to identify possible dynamical mechanisms behind the complex behavior of the whole network. Recently, the concept of winnerless competition networks has been introduced to investigate spatiotemporal encoding by an assembly of neurons [6]. To explore this concept, a network of FitzHugh-Nagumo spiking neurons coupled by time-dependent inhibitory interactions has been investigated to reveal how input information can be efficiently transformed into spatiotemporal firing patterns [6].

In this Letter the focus is on the behavior of a network made of simpler neuronal units. Each neuron is represented by a two-dimensional map of the kind studied recently by Rulkov [7]. Obviously, from the point of view of neuroscience, this dynamical system is merely a toy model but, nevertheless, it shows enough dynamical complexity to mimic the characteristic behavior of more involved neuron models. At the same time, it is simple enough to permit a detailed analysis of the mechanism behind its remarkable encoding capabilities. By using this model we have found spatiotemporal patterns of activation and inactivation of neuronal subgroups that are similar to the patterns obtained by using more complex models of neuronal behavior [6]. When isolated, each neuron in the network is described by the twodimensional map

$$
\begin{gathered}
x_{k+1}=f\left(x_{k}, y_{k}\right), \\
y_{k+1}=y_{k}-\mu\left(x_{k}+1\right)+\mu \sigma_{0},
\end{gathered}
$$

where $\mu$ is a parameter setting the time scale of the $y$ variable. In what follows, we will take $\mu=0.001$ so that the characteristic time scales of both variables are widely separated. The parameter $\sigma_{0}$ represents the external input acting on the neuron. In Eq. (1), $f(x, y)$ is a function of the form

$$
f(x, y)= \begin{cases}y+\alpha(1-x)^{-1}, & x \leq 0 \\ y+\alpha, & 0<x<\alpha+y, \\ -1, & x \geq \alpha+y\end{cases}
$$

where $\alpha$ is a characteristic parameter. In a neurobiological context, $x$ and $y$ are the fast and slow dynamical variables describing the behavior of the neuron and, in particular, the fast variable represents the instantaneous voltage across the neuron's membrane.

Rulkov has shown that this map has basically three dynamical regimes that are dependent on the values of the parameters $\alpha$ and $\sigma_{0}$ [7]. Specifically, the neuron can be silent or nonspiking (for small $\alpha$ and $\sigma_{0}$ ), in a regime of single spiking (for $\sigma_{0} \geq 0$ ) or in a spiking-bursting regime, where the neuron fires bursts of closely spaced spikes riding a slower wave. Let us consider this last case. Because of the smallness of the parameter $\mu$, the evolution of the fast variable $x$ can be analyzed by considering the variable $y$ as a slowly drifting control parameter. It follows from Eqs. (2) and (3) that the value of $y$ remains unchanged $\left(y_{k+1}=y_{k}\right)$ only if 


$$
x=x_{s}=-1+\sigma_{0} .
$$

Notice that if $x>x_{s}$, then the value of $y$ slowly increases upon iteration of the map, whereas if $x<x_{s}$, then $y$ decreases under the same circumstances. On the other hand, the functions giving the fixed points of the fast map can be obtained directly from Eq. (2),

$$
x=\frac{(1+y) \pm \sqrt{(1+y)^{2}-4(\alpha+y)}}{2}, \quad(x \leq 0) .
$$

This expression defines the stable $(s)$ and unstable $(u)$ branches of slow motion across the phase space. These branches have been plotted in Fig. 1. The dynamics of the system in the bursting regime can be analyzed in terms of the parameter $\sigma_{0}$. If the fixed point $x_{s}=-1+\sigma_{0}$ is on the stable branch, then the neuron is in a silent state. On the other hand, when $x_{s}$ is on the unstable branch, as shown in Fig. 1, the neuron is in the bursting regime. Notice that the slow motion along the stable branch of fixed points corresponds to the last part of the interburst intervals. This slow motion ends at the point where stable and unstable branches of fixed points meet and disappear. There, the phase point must jump to the spiking branch (not shown in Fig. 1). In doing so, the line $x=x_{s}$ is crossed from below and $y$ will subsequently decrease slowly as the phase point carries out the spiking part of the cycle. The burst ends at a homoclinic bifurcation that takes place at the crossing of the unstable branch with the line $x=-1$ [7]. On reaching this point, the phase point must jump back to the stable branch, where $y$ slowly increases with time, thus leading the phase point to move along $s$ and making the whole process to restart

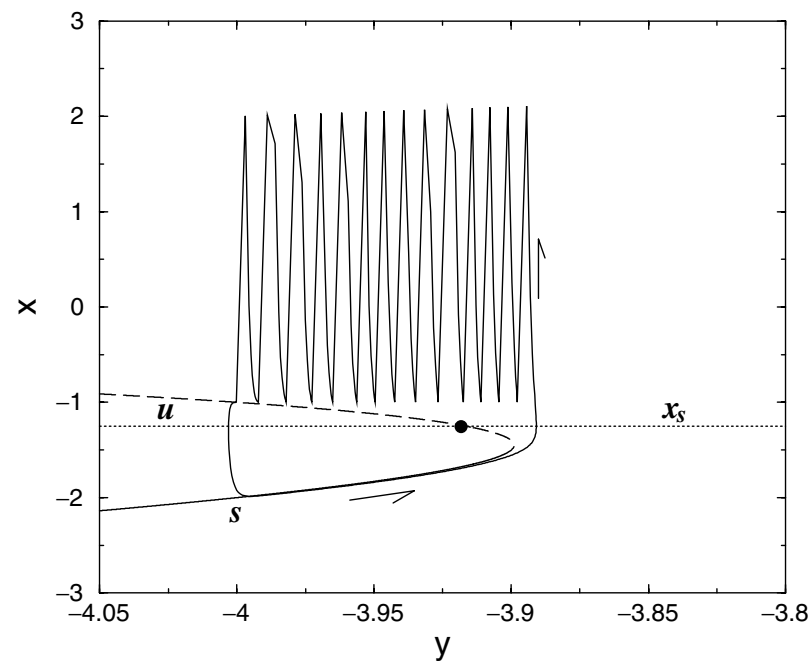

FIG. 1. Stable $(s)$ and unstable $(u)$ branches of the slow dynamics of one isolated neuron plotted on the phase plane $(y, x)$. The parameters are $\alpha=6$ and $\sigma_{0}=-0.25$. The temporal evolution of the phase point during the time course of a burst is also depicted to illustrate the discussion carried out in the text. itself. In Fig. 1 one of these bursts has been superimposed to the bifurcation diagram of the fast subsystem to illustrate the structure of the bursting oscillation.

Let us address the main topic of this Letter by considering a network of neurons described by the following set of $N$ coupled maps

$$
\begin{gathered}
x_{k+1}^{(n)}=f\left(x_{k}^{(n)}, y_{k}^{(n)}\right), \\
y_{k+1}^{(n)}=y_{k}^{(n)}-\mu\left(x_{k}^{(n)}+1\right)+\mu \sigma^{(n)}, \\
\sigma^{(n)}=\sigma_{0}^{(n)}-\sum_{m=1}^{N} g_{n, m}\left(x_{k}^{(m)}-\nu\right),
\end{gathered}
$$

where $n=1,2, \ldots, N$. Notice that, in principle, the external input $\sigma_{0}^{(n)}$ can be different for different neurons in the network. Part of the whole input acting on each neuron comes from its couplings to other units in the network. Here, the coefficient $g_{n, m}$ gives the action on neuron $n$ coming from neuron $m$. As the parameter $\nu$ is chosen to be smaller than the minimum value of all the $x^{(n)}$, the effect of the rest of the neurons on the dynamics of each one of them is purely inhibitory. In Fig. 2 we present the architecture of a network of nine neurons whose synchronizing behavior is going to be explored next.

Let us consider that the stimulus acting on each neuron is identical, that is, let us take $\sigma_{0}^{(n)}=\sigma_{0}$ for all $n$. The behavior of one coupled neuron can be analyzed by using the same argument employed for the isolated case. Let us consider, for example, the behavior of neuron 1. Its branches of stable and unstable fixed points are still given by Eq. (5) but now, the constancy of $y^{(1)}$ during the fast time scale leads to the condition

$$
\hat{x}_{k}^{(1)}=-1+\sigma_{0}-\left(x_{k}^{(2)}-\nu\right),
$$

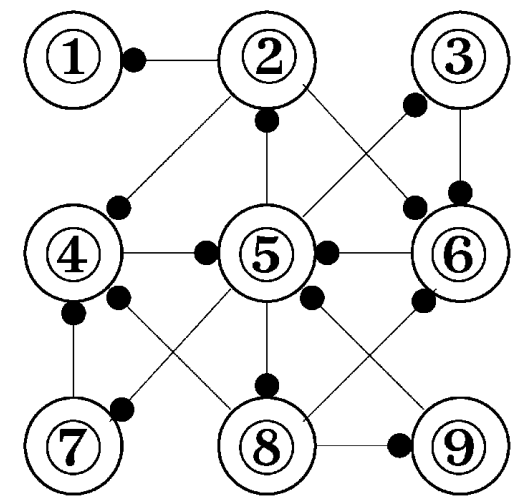

FIG. 2. The structure of a neural network with some neurons being connected through purely inhibitory synapses. Notice that $g_{n, m} \neq g_{m, n}$. The directional connections explicitly drawn by means of solid dots correspond to $g_{n, m}=1.0$. All the other terms in Eq. (8) take values $g_{n, m}=0$. 
instead of that given by Eq. (4). Now, the value of $x^{(1)}$ at which $y^{(1)}$ remains unchanged is no longer stationary but oscillates in time due to the motion of the variable $x^{(2)}$ and, in particular, it can be driven from the unstable to the stable branch of fixed points or vice versa. For some fixed values of $\sigma_{0}$, each burst of neuron 2 can drive $\hat{x}^{(1)}$ to cross the stable branch $s$ and then, neuron 1 is pushed to its silent regime. On the contrary, when neuron 2 is in the interval between two bursts, $\hat{x}^{(1)}$ is driven to cross the unstable branch $u$ and thus neuron 1 enters its fast spiking regime. Indeed, as we can see in Fig. 3, neuron 1 fires during the last part of the silent phase of neuron 2, that is, when the phase point of this last neuron is moving along its stable branch of fixed points. Notice that, when driven to excitation, neuron 1 must fire the burst entirely even if the driving provided by neuron 2 returns to subthreshold values. This argument explains why the successive bursts of neurons 1 and 2 are forced to take place at different times. Obviously, this same analysis applies to neurons driven by two or more inhibitory inputs although in those cases the behavior can be more complex due to the existence of closed feedback loops among the neurons. Thus, the degree of overlapping among the different bursts depends critically on the detailed architecture of the network.

As a whole, the network acts as a dynamical system having different regimes. If $\sigma_{0}$ is small enough, all neurons decay to their resting state after an initial transient. When the constant (tonic) stimulus is increased, some

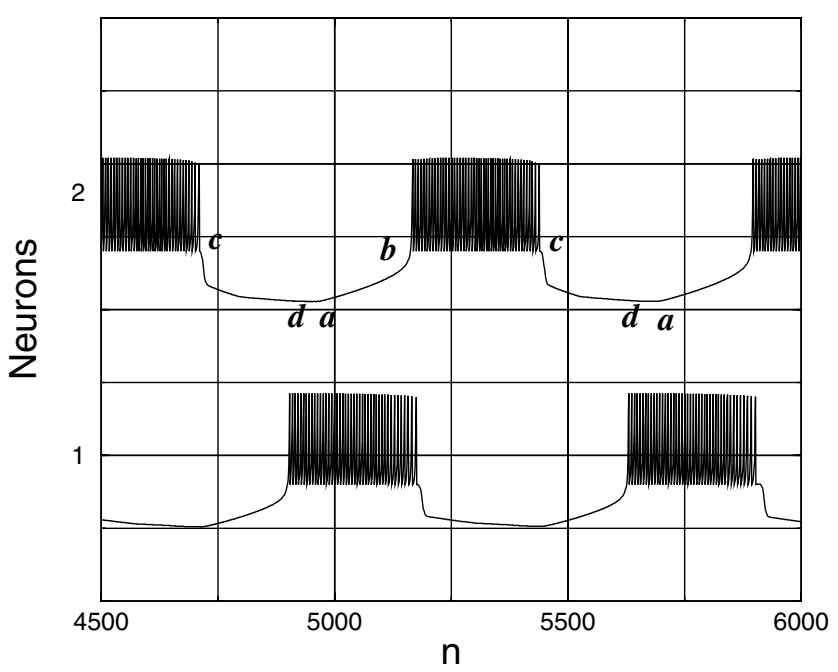

FIG. 3. Sequence of bursts generated by neurons 1 and 2 under the action of a suprathreshold stimulus of constant intensity. In $a$, neuron 2 is moving slowly along its stable branch of fixed points. Just a little earlier, in $d$, it has induced the firing of neuron 1 through the inhibitory coupling. Notice that, once started, the firing of neuron 1 must perform the whole burst even if neuron 2 returns quickly to subthreshold values. This leads to a small overlapping of the bursts fired by both neurons near the instant labeled $b$. groups of neurons start the synchronous firing of spikes, whereas others perform only a small slow-wave oscillatory behavior associated with the interburst time scale. When the stimulus is further increased until it reaches a given threshold ( $\sigma_{0} \approx-0.85$ ), all the neurons in the network start firing successive bursts of spikes. In Fig. 4 we present some bursts produced by a suprathreshold stimulus acting on all the neurons with the same intensity and timing. As we can observe, the network develops a spatiotemporal pattern of firing that amounts to the transient activation and inactivation of four different assemblies of neurons. In fact, neurons labeled 1 and 9 fire in synchrony and will be considered as assembly $A$. The same occurs with neurons labeled 2, 3, 7, and 8 (assembly $B$ ) and with neurons labeled 4 and 6 (assembly $C$ ). Assembly $D$ includes only the neuron labeled 5 . The activity of all the neurons belonging to the same assembly is synchronous not only at the level of bursts but also at the level of individual spikes. Notice that, in spite of the links connecting only neighboring neurons, the different assemblies can include also the not neighboring units. Thus, the existence of inhibitory couplings induces a global structure on the whole network.

The lowest signal depicted in Fig. 4 is the average of the spike trains fired by all the neurons belonging to the network

$$
(\mathrm{FP})_{k}=N^{-1} \sum_{n=1}^{N} x_{k}^{(n)}
$$

As we can see, this last signal seems to present a periodic variation with a characteristic frequency. However, this

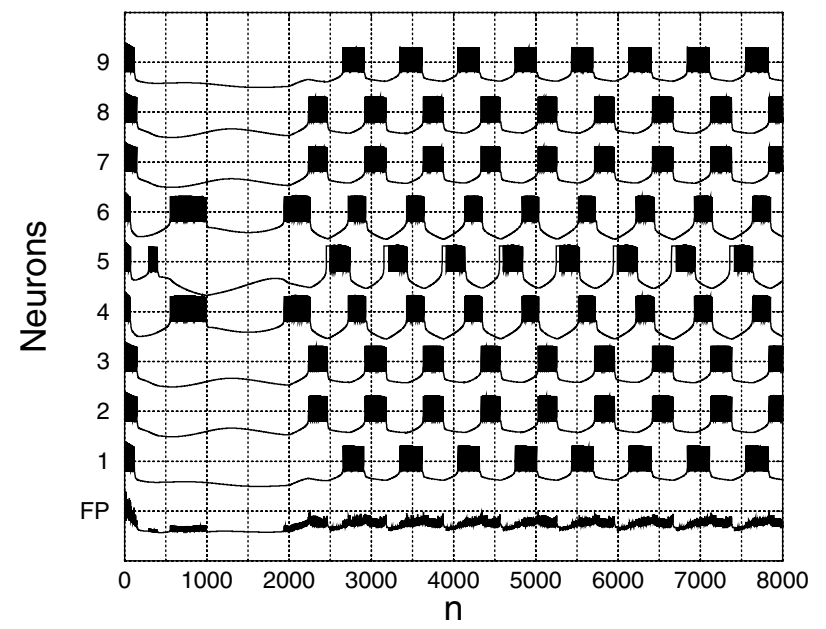

FIG. 4. Spike trains generated by the neurons of the network under the action of a suprathreshold stimulus of constant intensity. The stimulus is delivered to all neurons at $n=$ 2000 , and it consists in a sudden elevation of $\sigma_{0}$ from -2.0 to 0.0 , all the neurons being stimulated in the same way. The plot labeled FP has been obtained by averaging the instantaneous values of all the spike trains. 


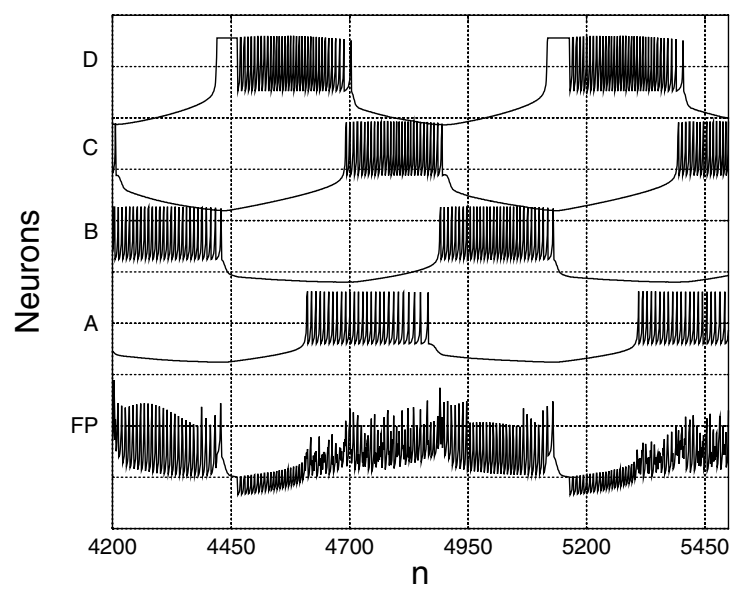

FIG. 5. The structure of the field potential oscillation as a consequence of the transient activation and inactivation of the four assemblies of neurons following the sequence $D-A-B-C-D-A-\ldots$ Each spike train in the figure represents one assembly of neurons firing synchronously. The contribution of each assembly to the FP signal depends on the number of neurons that constitute the assembly. The FP signal in this plot has been scaled up by a factor of 3 in order to depict more clearly its characteristic features.

signal is not exactly periodic in all its details due to variability displayed by the different bursts fired by each neuron. As the value of $\sigma_{0}$ is increased, the frequency of the bursts displayed by the field potential (FP) oscillation defined by Eq. (10) also increases, thus providing a kind of rate coding. The detailed form of this oscillatory FP signal comes from the transient overlapping of the successive bursts coming from the different assemblies. In Fig. 5 we can observe a close-up view of the temporal development of the firing across the network. Notice that during some epochs, the only contribution to the FP oscillation comes from the activity of a single assembly, and thus the global output of the network is synchronized by the rhythm of one of its subgroups. In other epochs the FP oscillation results from the weighted contributions of one, two, or even three different assemblies. It is clear that the whole temporal structure of the network's output will also depend on the location and intensity of the inputs. When stimulated differently, the synchronous activity found under identical driving no longer appears, and consequently the number of assemblies as well as the succession of bursts becomes altered with respect to the case of homogeneous stimulation.
The use of a simplified, yet far from trivial, neuron model has allowed us to build a winnerless competition network in which transient activation and inactivation of some groups of neurons appear as a consequence of pairwise inhibitory couplings between them. According to previous results obtained by using more complex model neurons, these transient local patterns seem to be generic in networks having deterministic trajectories connecting fixed points and limit cycles in the state space of the whole system. The model studied in this Letter proves that dynamical encoding appears also in networks where the dynamical complexity of each neuron model has been considerably reduced.

For these networks, the nature of the synchronization allows the different stimuli to be dynamically encoded by specific and reproducible sequences of firing coming from different assemblies of neurons across the network, a property that can be used to perform discrimination tasks. The simplicity of the model neuron used in this work allows a detailed analysis of the mechanisms behind the dynamical behavior of the network and, furthermore, it will permit the modeling and implementation of large networks (with hundreds of neurons, for example) by using a reasonable computing capacity.

The author acknowledges the Dirección General de Investigación Científica y Técnica (DGICYT) of Spain for support (Project No. BFM2002-03822).

*Electronic address: casado@us.es

[1] A. Scott, Neuroscience. A Mathematical Primer (Springer, New York, 2002).

[2] F. Rieke, D. Varland, R. de Ruyter van Steveninck, and W. Bialek, Spikes: Exploring the Neural Code (MIT Press, Cambridge, MA, 1997).

[3] M. Wehr and G. Laurent, Nature (London) 384, 162 (1996).

[4] M. Bazhenov, M. Stopfer, M. Rabinovich, H. D. I. Abarbanel, T. J. Sejnowski, and G. Laurent, Neuron 30, 569 (2001).

[5] M. Bazhenov, M. Stopfer, M. Rabinovich, R. Huerta, H. D. I. Abarbanel, T. J. Sejnowski, and G. Laurent, Neuron 30, 553 (2001).

[6] M. Rabinovich, A. Volkovskii, P. Lecanda, R. Huerta, H. D. I. Abarbanel, and G. Laurent, Phys. Rev. Lett. 87, 068102 (2001).

[7] N. F. Rulkov, Phys. Rev. E 65, 041922 (2002). 